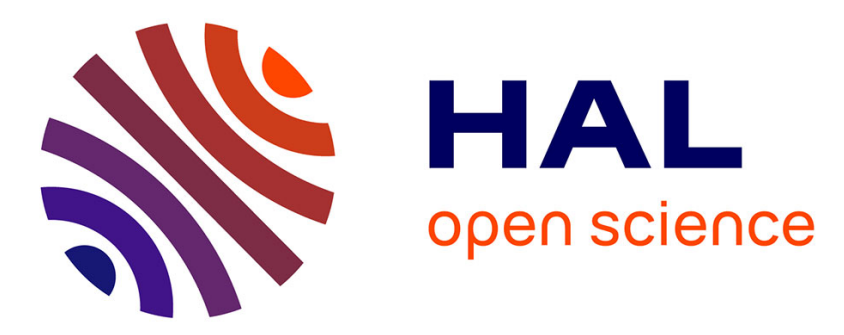

\title{
WAVE FINITE ELEMENT METHOD FOR COMPUTING THE DYNAMIC RESPONSE OF RAILWAY TRANSITION ZONES SUBJECTED TO MOVING LOADS
}

Benjamin Claudet, Tien Hoang, Denis Duhamel, Gilles Forêt, Jean-Luc L Pochet, Francis Sabatier

\section{To cite this version:}

Benjamin Claudet, Tien Hoang, Denis Duhamel, Gilles Forêt, Jean-Luc L Pochet, et al.. WAVE FINITE ELEMENT METHOD FOR COMPUTING THE DYNAMIC RESPONSE OF RAILWAY TRANSITION ZONES SUBJECTED TO MOVING LOADS. COMPDYN 2019, Jun 2019, Crete, Greece. hal-02915351

\author{
HAL Id: hal-02915351 \\ https://hal.science/hal-02915351
}

Submitted on 14 Aug 2020

HAL is a multi-disciplinary open access archive for the deposit and dissemination of scientific research documents, whether they are published or not. The documents may come from teaching and research institutions in France or abroad, or from public or private research centers.
L'archive ouverte pluridisciplinaire HAL, est destinée au dépôt et à la diffusion de documents scientifiques de niveau recherche, publiés ou non, émanant des établissements d'enseignement et de recherche français ou étrangers, des laboratoires publics ou privés. 


\title{
WAVE FINITE ELEMENT METHOD FOR COMPUTING THE DYNAMIC RESPONSE OF RAILWAY TRANSITION ZONES SUBJECTED TO MOVING LOADS
}

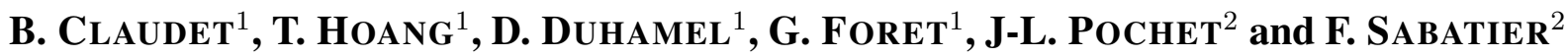 \\ ${ }^{1}$ Laboratoire Navier, UMR 8205, Ecole des Ponts ParisTech \\ IFFSTTAR, CNRS, UPE, Champs-sur-Marne, France \\ e-mail: \{benjamin.claudet, tien.hoang, denis.duhamel, gilles.foret\}@enpc.fr \\ 2 Eurotunnel \\ BP69, 62904 Coquelles Cedex, France \\ e-mail: \{franncis.sabatier, jean-luc.pochet\}@eurotunnel.com
}

Keywords: Wave Finite Element, Transition zones, Railway, Dynamics, Moving loads

\begin{abstract}
The dynamics of a railway track on transition zones is the subject of numerous analytical and numerical researches. This article presents a new development of the wave finite element method for such a structure in order to reduce the calculation time. We consider a transition zone which is between two half infinite periodic tracks (which could be of different types, e.g. a zone between ballasted and non-ballasted tracks) and train loads are represented by concentrated moving loads. We applied WFE for each half periodic track to obtain the relationship that links the responses of the boundary zone of each periodic part with the help of the wave analysis. Then, this relationship is combined with the dynamic equation of the zone to get the global dynamic equation of the whole railway track. This technique permits to reduce the degrees of freedom ( $D o F)$ of the track to those of the transition zone. Numerical studies show the influence of the geometrical and mechanical parameters of the transition zone on the mechanical response of the track with a transition zone compared to a uniform track.
\end{abstract}




\section{Introduction}

Mechanical behaviour of railway tracks have been studied for a long time. The most common model considers the track as an infinitely long beam resting on equally spaced identical supports. The dynamics of the track under some known loads can be solved analytically by modelling each support by a mass-springs-dampers system and the rail by an Euler beam [1-4] or a Timoshenko beam [5]. Metrikine and Popp [6] also modelled the support and the ballast - for ballasted tracks - as continuous media. All these studies used the Floquet's theorem which takes into account the spatial periodicity of the structure. In the case of transition zones, the structure is not periodic so the Floquet's theorem can't be used.

The high speed transition zone between a ballast to ballastless track situated in Chauconin (France) had been damaged by train traffic. These damages include hanging sleepers. Consequently, the train speed in this zone has been reduced from $320 \mathrm{~km} \cdot \mathrm{h}^{-1}$ to $230 \mathrm{~km} \cdot \mathrm{h}^{-1}$. This transition zone is the subject of both experimental and numerical studies [7, 8]. Fortunato et al. [9] also pointed out hanging sleepers which are due to the deformation of the lowest vertical stiffness part in transition zones. Wang [10] gave a review of studies dealing with transition zones in the railway domain. The problems encountered points out a need to better understand transition zones dynamics.

A default or a damage also breaks the track periodicity even if this default is very localised. Hoang et al. [11] studied the mechanical behaviour of such a track considering a constant moving load on a periodically supported beam. Considering a "sufficiently large" computation domain, Hoang et al. gave a digital method to compute this dynamic behaviour. Kouroussis et al. [12] proposed a two steps method for a numerical computation of the rail-support-train system response and applied it to some common defaults.

Dividing the structure into substructures and considering the equilibrium relationship in a substructure, Germonpré et al. [13] studied the free wave modes in a substructure and then computed the whole structure dynamics. By separating left going and right going modes, Germonpré et al. [14] applied this method to a transition zone including a train-track-soil coupling. Arlaud [15] developed a similar method excepted that it fixed the wave-number instead of fixing the frequency. Then, a mode reduction was performed in order to increase the calculation efficiency.

Like the method developed by Germonpré et al. [13], the wave finite element (WFE) method is a computational method for periodic structures and waveguides. In the WFE method, for each frequency, the equilibrium relationship in one pattern of the structure is transformed into a recurrence relationship. Free wave modes of the recurrence operator calculation is described in [16]. Hoang et al. improved this method by adding the possibility to take into account a load which may be applied to any pattern of the structure [17] and used it for computation of railway tracks dynamics [18].

In this article, a transition zone is defined as a zone between two semi-infinite periodic structures which can be different or not. This can represent either a zone passing from ballast to ballastless track or a damaged zone between two healthy identical zones. After this introduction, the classical WFE method is presented. The third part of this article presents new developments enabling the computation of transition zones mechanical behaviour. The forth part gives two examples of results of this method on the mechanical behaviour: effects of a damaged zone in an infinite periodic healthy track and effects of a variation in the support spacing. Then, the conclusions of this work are given in the last part. 


\section{WFE Method: Homogeneous Periodic Structure}

In this part an homogeneous periodic structure is considered. It is assumed that all the loads applied to the structure are known. The dynamic equation of one pattern (a substructure) at a given angular frequency $\omega$ is given by the equation (1), where $q$ is the vector containing the degrees of freedom of one substructure and $\mathbf{F}$ the vector containing the external loads applied on this substructure.

$$
\mathbf{D}_{t o t} \mathbf{q}=\mathbf{F}
$$

The dynamic stiffness matrix (DSM) $\mathbf{D}_{\text {tot }}$ can be calculated using the finite element method with $\mathbf{D}_{t o t}=\mathbf{K}+j \omega \mathbf{C}-\omega^{2} \mathbf{M}$ where $\mathbf{K}$ is the static stiffness matrix, $\mathbf{M}$ the mass matrix and $\mathrm{C}$ the damping matrix. Then, (1) can be rewritten separating the nodes of the left and right boundaries, and the inner nodes of the substructure respectively denoted with the subscripts $L, R$ and $I_{I}$. Hence, the relationship (2) is obtained.

$$
\left[\begin{array}{ccc}
\tilde{\mathbf{D}}_{I I} & \tilde{\mathbf{D}}_{I L} & \tilde{\mathbf{D}}_{I R} \\
\tilde{\mathbf{D}}_{L I} & \tilde{\mathbf{D}}_{L L} & \tilde{\mathbf{D}}_{L R} \\
\tilde{\mathbf{D}}_{R I} & \tilde{\mathbf{D}}_{R L} & \tilde{\mathbf{D}}_{R R}
\end{array}\right]\left[\begin{array}{c}
\mathbf{q}_{I} \\
\mathbf{q}_{L} \\
\mathbf{q}_{R}
\end{array}\right]=\left[\begin{array}{c}
\mathbf{F}_{I} \\
\mathbf{F}_{L} \\
\mathbf{F}_{R}
\end{array}\right]
$$

As shown in [18], this relation leads to the recurrence equation (3) where the superscript ${ }^{(n)}$ denotes for the $n^{\text {th }}$ substructure.

$$
\mathbf{u}^{(n+1)}=\mathbf{S} \mathbf{u}^{(n)}+\mathbf{b}^{(n)}
$$

where

$$
\mathbf{u}^{(n)}=\left[\begin{array}{c}
\mathbf{q}_{L}^{(n)} \\
-\mathbf{F}_{L}^{(n)}
\end{array}\right], \quad \mathbf{b}^{(n)}=\left[\begin{array}{c}
\mathbf{D}_{q I} \mathbf{F}_{I}^{(n)} \\
\mathbf{D}_{f I} \mathbf{F}_{I}^{(n)}-\mathbf{F}_{\partial R}^{(n)}
\end{array}\right]
$$

$\mathbf{F}_{\partial R}^{(n)}$ is the external load applied at the right boundary of the $n^{t h}$ substructure and $\mathbf{S}, \mathbf{D}_{q I}$ and $\mathbf{D}_{f I}$ definitions are given in [18].

That leads to the equation system (4):

$$
\left\{\begin{array}{l}
\mathbf{u}^{(n)}=\mathbf{S}^{n} \mathbf{u}^{(0)}+\sum_{k=1}^{n} \mathbf{S}^{n-k} \mathbf{b}^{(k-1)} \\
\mathbf{u}^{(-n)}=\mathbf{S}^{-n} \mathbf{u}^{(0)}-\sum_{k=1}^{n} \mathbf{S}^{-n+k-1} \mathbf{b}^{(-k)}
\end{array}\right.
$$

To compute the power of the $\mathbf{S}$ matrix, the eigenvalues and eigenvectors $\left\{\mu_{j}, \phi_{j}\right\}_{j}$ of the $\mathbf{S}$ matrix are computed by a $\mathbf{S}+\mathbf{S}^{-1}$ transformation. The eigenvalues come in pair $\left(\mu_{j}, \mu_{j}^{\star}\right)$ with $\left\|\mu_{j}\right\| \leqslant 1$ and $\mu_{j}^{\star}=\frac{1}{\mu_{j}}[16]$. The corresponding eigenvectors are noted $\left(\phi_{j}, \phi_{j}^{\star}\right)$. Let the eigenbasis $\left\{\boldsymbol{\Phi} \Phi^{\star}\right\}$ be defined as: $\boldsymbol{\Phi}=\left[\phi_{1} \ldots \phi_{n}\right]$ and $\boldsymbol{\Phi}^{\star}=\left[\phi_{1}^{\star} \ldots \phi_{n}^{\star}\right]$. Where the subscript " " (respectively " $F ")$ denotes for the components of this basis corresponding to the degrees of freedom (DoF) ${ }^{1}$ (resp. the loads), leading to (5).

$$
\boldsymbol{\Phi}=\left[\begin{array}{c}
\boldsymbol{\Phi}_{q} \\
\boldsymbol{\Phi}_{F}
\end{array}\right] \quad \boldsymbol{\Phi}^{\star}=\left[\begin{array}{c}
\boldsymbol{\Phi}_{q}^{\star} \\
\boldsymbol{\Phi}_{F}^{\star}
\end{array}\right]
$$

\footnotetext{
${ }^{1}$ Usually displacements/rotations
} 
In (6), $\mathbf{u}^{(n)}$ and $\mathbf{b}^{(n)}$ are written in this wave basis:

$$
\begin{aligned}
\mathbf{u}^{(n)} & =\boldsymbol{\Phi} \mathbf{Q}^{(n)}-\boldsymbol{\Phi}^{\star} \mathbf{Q}^{\star(n)} \\
\mathbf{b}^{(n)} & =\boldsymbol{\Phi} \mathbf{Q}_{E}^{(n)}-\boldsymbol{\Phi}^{\star} \mathbf{Q}_{E}^{\star(n)}
\end{aligned}
$$

Hoang et al. proved [18]:

$$
\begin{aligned}
& \mathbf{Q}_{E}^{(k)}=\left[\left(\boldsymbol{\mu} \boldsymbol{\Phi}_{q}^{\star T} \mathbf{D}_{L I}+\boldsymbol{\Phi}_{q}^{\star T} \mathbf{D}_{R I}\right) \mathbf{F}_{I}^{(k)}-\boldsymbol{\Phi}_{q}^{\star T} \mathbf{F}_{\partial R}^{(k)}\right] \\
& \mathbf{Q}_{E}^{\star(k)}=\left[\left(\boldsymbol{\mu}^{\star} \boldsymbol{\Phi}_{q}^{T} \mathbf{D}_{L I}+\boldsymbol{\Phi}_{q}^{T} \mathbf{D}_{R I}\right) \mathbf{F}_{I}^{(k)}-\boldsymbol{\Phi}_{q}^{T} \mathbf{F}_{\partial R}^{(k)}\right]
\end{aligned}
$$

\section{Transition Zone between Two Semi-infinite Zones}

In this part, a transition zone between two infinite periodic zones (see figure 1) is considered. For railways the transition zone can for instance be a zone between a ballasted track and a concrete slab track. These two types of track have different stiffness. Therefore, $\mathbf{S}$ can be different in each zone and some formulas from the previous part are no longer valid.

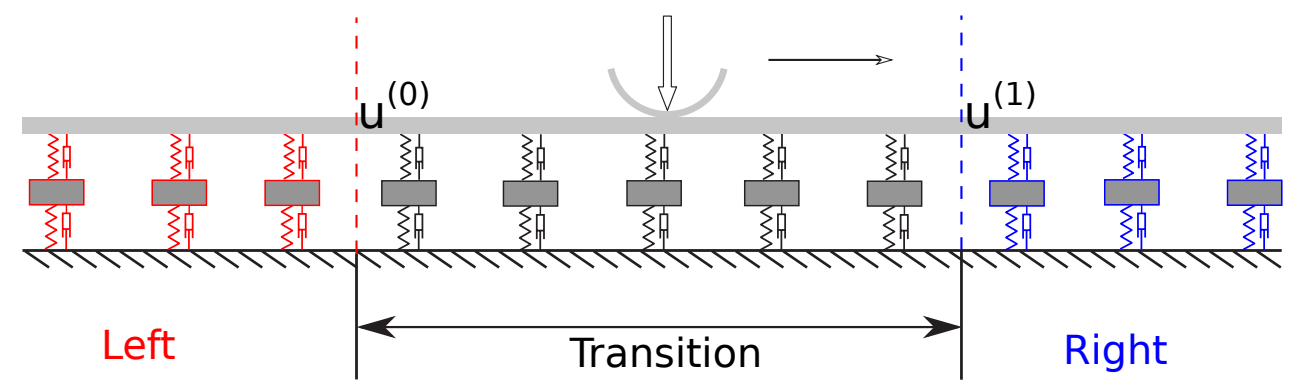

Figure 1: Transition zone between two semi-infinite periodic railway tracks.

In the transition zone the equilibrium relationship is:

$$
\mathbb{D}_{H, \text { trans }}\left[\begin{array}{c}
\mathbf{q}_{I} \\
\mathbf{q}_{L} \\
\mathbf{q}_{R}
\end{array}\right]=\left[\begin{array}{c}
\mathbf{F}_{I} \\
\mathbf{F}_{L} \\
\mathbf{F}_{R}
\end{array}\right]
$$

Where $\mathbb{D}_{H, \text { trans }}$ is the dynamic stiffness matrix (DSM) of the transition zone.

The equilibrium relationships for one pattern in the left and right zones were written in the same way replacing $\mathbb{D}_{H, \text { trans }}$ by the DSM of the corresponding zone. So, applying the same method as in the previous part, propagation equations (9) were obtained (instead of (3)) - with $"{ }_{R} "$ subscript denoting for the right zone and " $L "$ subscript the left zone.

$$
\left\{\begin{array}{l}
\forall n \geqslant 1, \mathbf{u}_{R}^{(n+1)}=\mathbf{S}_{R} \mathbf{u}^{(n)}+\mathbf{b}^{(n)} \\
\forall n \leqslant 0, \mathbf{u}_{L}^{(n-1)}=\mathbf{S}_{L}^{-1} \mathbf{u}^{(n)}-\mathbf{S}_{L}^{-1} \mathbf{b}^{(n-1)}
\end{array}\right.
$$

With $\mathbf{u}^{(0)}$ corresponding to the left boundary of the transition zone, and $\mathbf{u}^{(1)}$ to the left boundary of the right zone (see figure 1 ). 


\subsection{Decomposition in the Right Zone and in the Left Zone}

Thus, for the right zone the recurrence relationship is:

$$
\forall n \geqslant 1, \mathbf{u}^{(n)}=\mathbf{S}_{R}^{n-1} \mathbf{u}^{(1)}+\sum_{k=2}^{n} \mathbf{S}_{R}^{n-k} \mathbf{b}^{(k-1)}
$$

Let $\mathbf{u}_{R}=\mathbf{u}^{(1)}$. As in the first part, $\mathbf{u}_{R}$ was rewritten in the eigenbasis $\left\{\mathbf{Q}_{R}, \mathbf{Q}_{R}^{\star}\right\}$ of $\mathbf{S}_{R}$ associated with the eigenvalues $\left\{\boldsymbol{\mu}_{R}, \boldsymbol{\mu}_{R}^{\star}\right\}$ where $\left\|\mu_{R, j}\right\| \leqslant 1$ and $\left\|\mu_{R, j}^{\star}\right\| \geqslant 1$. That led to the wave decomposition (11) for $\mathbf{u}_{R}$ and (12) for $\mathbf{u}^{(n)}$ and $\mathbf{b}^{(n)}$ :

$$
\begin{gathered}
\mathbf{u}_{R}=\boldsymbol{\Phi}_{R} \mathbf{Q}_{R}-\boldsymbol{\Phi}_{R}^{\star} \mathbf{Q}_{R}^{\star} \\
\forall n \geqslant 1,\left\{\begin{array}{l}
\mathbf{u}^{(n)}=\boldsymbol{\Phi}_{R} \mathbf{Q}^{(n)}-\boldsymbol{\Phi}_{R}^{\star} \mathbf{Q}^{\star(n)} \\
\mathbf{b}^{(n)}=\boldsymbol{\Phi}_{R} \mathbf{Q}_{E}^{(n)}-\boldsymbol{\Phi}_{R}^{\star} \mathbf{Q}_{E}^{\star(n)}
\end{array}\right.
\end{gathered}
$$

Using (10) with (11) and (12) led to:

$$
\begin{aligned}
\mathbf{u}^{(n)} & =\boldsymbol{\Phi}_{R} \mathbf{Q}^{(n)}-\boldsymbol{\Phi}_{R}^{\star} \mathbf{Q}^{\star(n)} \\
& =\boldsymbol{\Phi}_{R} \boldsymbol{\mu}_{R}^{n-1}\left(\mathbf{Q}_{R}+\sum_{k=2}^{n} \boldsymbol{\mu}_{R}^{\star k-1} \mathbf{Q}_{E}^{(k-1)}\right)-\boldsymbol{\Phi}_{R}^{\star} \boldsymbol{\mu}_{R}^{\star n-1}\left(\mathbf{Q}_{R}^{\star}+\sum_{k=2}^{n} \boldsymbol{\mu}_{R}^{k-1} \mathbf{Q}_{E}^{(k-1)}\right)
\end{aligned}
$$

$\left\|\boldsymbol{\mu}_{R}^{\star}\right\|>1$, so taking the limit $n \rightarrow+\infty$ led to $\mathbf{Q}_{R}^{\star}=-\sum_{k=2}^{+\infty} \boldsymbol{\mu}_{R}^{k-1} \mathbf{Q}_{E}^{(k-1)}$ because DoF and loads can't be infinite. (13) then became (14).

$$
\left\{\begin{array}{r}
\mathbf{q}_{R}=\boldsymbol{\Phi}_{R, q} \mathbf{Q}_{R}+\boldsymbol{\Phi}_{R, q}^{\star} \sum_{k=1}^{\infty} \boldsymbol{\mu}_{R}^{k} \mathbf{Q}_{E}^{\star(k)} \\
\mathbf{F}_{R}-\mathbf{F}_{\partial R}=\boldsymbol{\Phi}_{R, F} \mathbf{Q}_{R}+\boldsymbol{\Phi}_{R, F}^{\star} \sum_{k=1}^{\infty} \boldsymbol{\mu}_{R}^{k} \mathbf{Q}_{E}^{\star(k)}
\end{array}\right.
$$

With the same reasoning on the left part of the structure, the relationship (15) was proved.

$$
\left\{\begin{array}{r}
\mathbf{q}_{L}=\boldsymbol{\Phi}_{L, q} \sum_{k=1}^{\infty} \boldsymbol{\mu}_{L}^{k-1} \mathbf{Q}_{E}^{(-k)}-\boldsymbol{\Phi}_{L, q}^{\star} \mathbf{Q}_{L}^{\star} \\
-\mathbf{F}_{L}=\boldsymbol{\Phi}_{L, F} \sum_{k=1}^{\infty} \boldsymbol{\mu}_{L}^{k-1} \mathbf{Q}_{E}^{(-k)}-\boldsymbol{\Phi}_{L, F}^{\star} \mathbf{Q}_{L}^{\star}
\end{array}\right.
$$

Finally, by substituting (14) and (15) into $(8),(16)$ was obtained:

$$
\begin{aligned}
\mathbb{D}_{H, \text { trans }}\left[\begin{array}{c}
\mathbf{q}_{I} \\
-\boldsymbol{\Phi}_{L, q}^{\star} \mathbf{Q}_{L}^{\star} \\
\boldsymbol{\Phi}_{R, q} \mathbf{Q}_{R}
\end{array}\right]+\mathbb{D}_{H, \text { trans }} & {\left[\begin{array}{c}
\mathbf{0} \\
\boldsymbol{\Phi}_{L, q} \sum_{k=1}^{\infty} \boldsymbol{\mu}_{L}^{k-1} \mathbf{Q}_{E}^{(-k)} \\
\boldsymbol{\Phi}_{R, q}^{\star} \sum_{k=1}^{\infty} \boldsymbol{\mu}_{R}^{k} \mathbf{Q}_{E}^{\star(k)}
\end{array}\right]=} \\
& {\left[\begin{array}{c}
\mathbf{0} \\
\mathbf{\Phi}_{L, F}^{\star} \mathbf{Q}_{L}^{\star} \\
\boldsymbol{\Phi}_{R, F} \mathbf{Q}_{R}
\end{array}\right]+\left[\begin{array}{c}
-\boldsymbol{\Phi}_{L, F} \sum_{k=1}^{\infty} \boldsymbol{\mu}_{L}^{k-1} \mathbf{Q}_{E}^{(-k)} \\
\mathbf{F}_{\partial R}+\mathbf{\Phi}_{R, F}^{\star} \sum_{k=1}^{\infty} \boldsymbol{\mu}_{R}^{k} \mathbf{Q}_{E}^{\star(k)}
\end{array}\right] }
\end{aligned}
$$


Which was rewritten as follows:

$$
\left(\mathbb{D}_{H, \text { trans }} \mathbb{C}_{q}-\mathbb{C}_{F}\right) \tilde{\mathbf{q}}=\tilde{\mathbf{F}}
$$

where

$$
\begin{array}{r}
\tilde{\mathbf{q}}=\left[\begin{array}{c}
\mathbf{q}_{I} \\
\mathbf{Q}_{L}^{\star} \\
\mathbf{Q}_{R}
\end{array}\right], \mathbb{C}_{q}=\left[\begin{array}{ccc}
\mathbf{I} & \mathbf{0} & \mathbf{0} \\
\mathbf{0} & -\boldsymbol{\Phi}_{L, q}^{\star} & \mathbf{0} \\
\mathbf{0} & \mathbf{0} & \boldsymbol{\Phi}_{R, q}
\end{array}\right], \mathbb{C}_{F}=\left[\begin{array}{ccc}
\mathbf{0} & \mathbf{0} & \mathbf{0} \\
\mathbf{0} & \boldsymbol{\Phi}_{L, F}^{\star} & \mathbf{0} \\
\mathbf{0} & \mathbf{0} & \boldsymbol{\Phi}_{R, F}
\end{array}\right] \\
\tilde{\mathbf{F}}=\left[\begin{array}{c}
\mathbf{F}_{I} \\
-\boldsymbol{\Phi}_{L, F} \sum_{k=1}^{\infty} \boldsymbol{\mu}_{L}^{k-1} \mathbf{Q}_{E}^{(-k)} \\
\mathbf{F}_{\partial R}+\boldsymbol{\Phi}_{R, F}^{\star} \sum_{k=1}^{\infty} \boldsymbol{\mu}_{R}^{k} \mathbf{Q}_{E}^{\star(k)}
\end{array}\right]-\mathbb{D}_{H}\left[\begin{array}{c}
\mathbf{0} \\
\boldsymbol{\Phi}_{L, q} \sum_{k=1}^{\infty} \boldsymbol{\mu}_{L}^{k-1} \mathbf{Q}_{E}^{(-k)} \\
\boldsymbol{\Phi}_{R, q}^{\star} \sum_{k=1}^{\infty} \boldsymbol{\mu}_{R}^{k} \mathbf{Q}_{E}^{\star(k)}
\end{array}\right]
\end{array}
$$

In the case of a constant moving load $2, \mathbf{Q}_{E}^{\star(k)}$ and $\mathbf{Q}_{E}^{(-k)}$ follow:

$$
\forall k \geqslant 1,\left\{\begin{array}{l}
\mathbf{Q}_{E}^{\star(k)}=e^{-i(k-1) \frac{\omega L}{v}} \mathbf{Q}_{E}^{\star(1)} \\
\mathbf{Q}_{E}^{(-k)}=e^{i(k-1) \frac{\omega L}{v}} \mathbf{Q}_{E}^{(-1)}
\end{array}\right.
$$

That led to (20):

$$
\left\{\begin{array}{l}
\sum_{k=1}^{\infty} \boldsymbol{\mu}_{L}^{k-1} \mathbf{Q}_{E}^{(-k)}=\frac{1}{1-\boldsymbol{\mu}_{L} e^{i \frac{\omega L}{v}}} \mathbf{Q}_{E}^{(-1)} \\
\sum_{k=1}^{\infty} \boldsymbol{\mu}_{R}^{k} \mathbf{Q}_{E}^{\star(k)}=\frac{\boldsymbol{\mu}_{R}}{1-\boldsymbol{\mu}_{R} e^{-i \frac{\omega L}{v}}} \mathbf{Q}_{E}^{\star(1)}
\end{array}\right.
$$

With $\mathbf{Q}_{E}^{\star(k)}$ and $\mathbf{Q}_{E}^{(k)}$ given by (7).

Then, $\tilde{\mathbf{q}}$ can be computed by inverting the linear equation (17). This computation gives the DoF of each node in the transition zone $\left(\mathbf{q}_{I}\right)$ and at its boundaries $\left(\mathbf{q}_{L}\right.$ and $\mathbf{q}_{R}$ calculated with (15) and (14)). The DoF in the other zones can be calculated using the classical WFE method (ie. using (10) for the right zone and a similar formula for the left zone).

\section{Examples}

In this part, the track considered is a ballastless track in which each support contains two elastic floors: one under rail rubber pad and one under sleeper rubber pad. The support was modelled as a mass (the sleeper) between two damper-spring systems (the pads). B21 elements from ABAQUS were used to get the beam DSM and the supports dynamic stiffness were added to the corresponding terms to obtain the DSM of each substructure. The beam cell length was $1 \mathrm{~cm}$. In the results presented here, the spatial period choosen for the computation was of the same length $L=3 \mathrm{~m}$ for each zone (five sleepers per period). The right, left and transition zones are represented in the figure 1 . To simplify results understanding, only a single load moving at a constant speed was applied. Table 1 gives the rail and supports mechanical characteristics and the load characteristics.

\subsection{Periodically Supported Beam with a Broken Support}

We considered a periodically supported beam resting on identical supports which means that the right and left zones were identical. To model a broken support, we assumed that this support had no influence on the structure stiffness. This is equivalent to a lack of one support. This broken support was located in the middle of the transition zone $(x=0 \mathrm{~m})$.

\footnotetext{
${ }^{2}$ 19] remains true if the load is repeated with a spatial period equal to the length of one substructure.
} 


\begin{tabular}{llll}
\hline Content & Notation & Value & $\mathrm{Unit}$ \\
\hline Rail linear mass & $\rho S$ & 60 & $\mathrm{~kg} \cdot \mathrm{m}^{-1}$ \\
Load speed & $v$ & 38 & $\mathrm{~m} \cdot \mathrm{s}^{-1}$ \\
Load applied & $Q$ & 100 & $\mathrm{kN}$ \\
Rail stiffness & $E I$ & 6.38 & $\mathrm{MN} \cdot \mathrm{m}^{2}$ \\
Sleeper mass & $m$ & 100 & $\mathrm{~kg}$ \\
Sleeper spacing & $l$ & 0.6 & $\mathrm{~m}$ \\
Under rail pad stiffness & $k_{1}$ & 192 & $\mathrm{MN} \cdot \mathrm{m}^{-1}$ \\
Under rail pad damping coefficient & $\eta_{1}$ & 1.97 & $\mathrm{MN} \cdot \mathrm{s} \cdot \mathrm{m}^{-1}$ \\
Under sleeper pad stiffness & $k_{2}$ & 26.4 & $\mathrm{MN} \cdot \mathrm{m}^{-1}$ \\
Under sleeper pad damping coefficient & $\eta_{2}$ & 0.17 & $\mathrm{MN} \cdot \mathrm{s} \cdot \mathrm{m}^{-1}$ \\
\hline
\end{tabular}

Table 1: Parameters of the load and railway track considered.

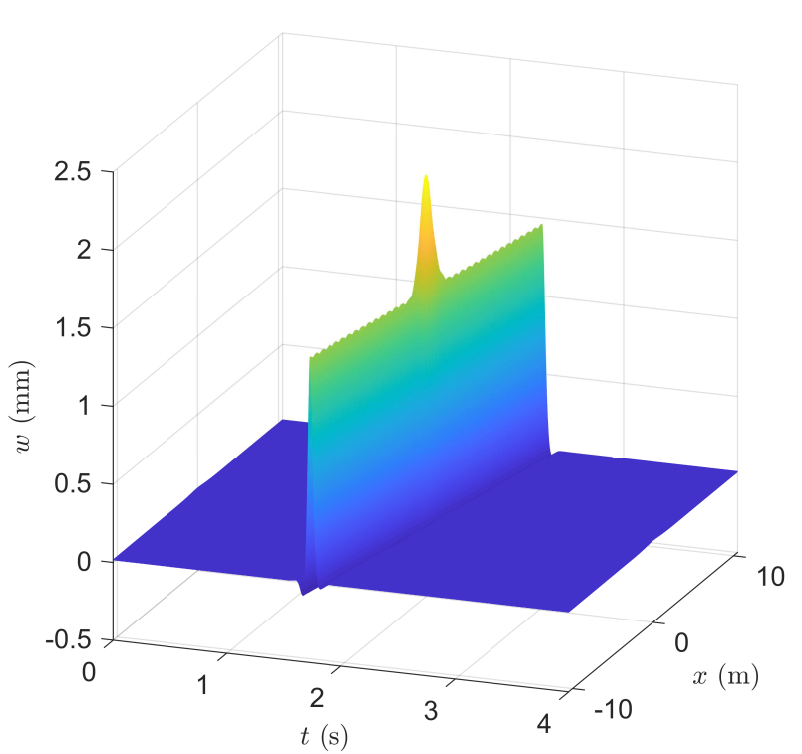

(a) Rail deflection against time and position.

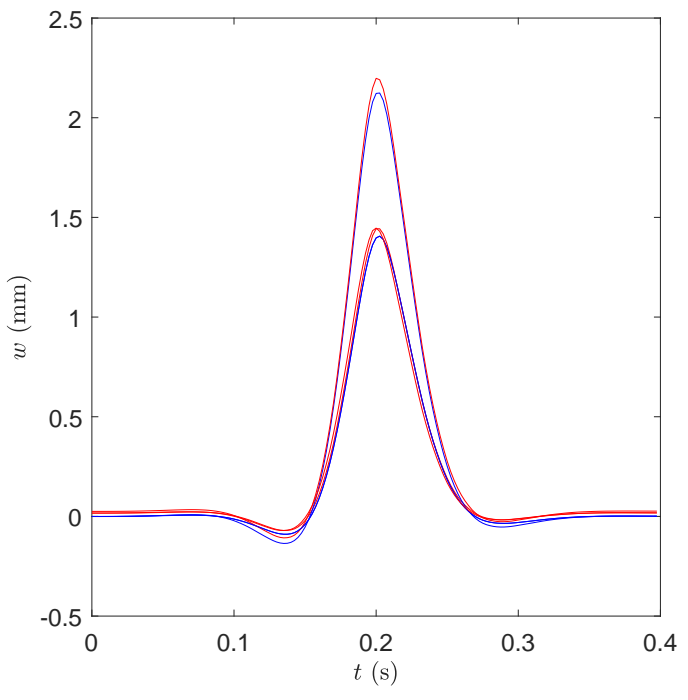

(b) Rail deflection against time for three different sleepers. WFE results (red curves) and results obtained with the method of [11] (blue curves).

Figure 2: Rail deflection for a track with a broken support.

Figure 2 shows the track deflection due to the load for this type of track. On the left side (figure 2a) the deflection is plotted against time and position. As the WFE method works in the frequency domain, an inverse Fourier transform was necessary to obtain temporal values. At each position, the maximum of the deflection corresponds to the time when the load is over this position. Consequently, the line of the maxima represents the load trajectory. As expected, the deflection is maximum for the broken support. On the right part of this figure (figure $2 b$ ), the deflection obtained by the WFE method (red curves) is compared with the one obtained with [11] method (blue curves, 21 supports modelled, 80 iterations). In this graph the deflection is plotted against time for three supports: the broken one $(x=0 \mathrm{~m})$ and two suports at $x= \pm 6 \mathrm{~m}$. These results show a good agreement which validated the WFE method for computing track deflection in damaged zones. 


\subsection{Variation of the Support Spacing}

In this example, the influence of a variation in the support spacing on the track behaviour was evaluated. In the left zone, the distance between two consecutive sleepers was $l_{L}=0,3 \mathrm{~m}$ whereas in right and transition zones, this distance was $l_{R}=l_{T}=0,6 \mathrm{~m}$. All the other parameters remained identical to the values given in the table 1 .

The computed deflection of this track is given in figure 3 . Once again, in the left part of the figure (figure 3a), the deflection is plotted against time and position. There were two times more support in the left zone, thus, as expected, this zone seems approximately two times stiffer. The right part of the figure (figure 3a gives the deflection at the frequency $5 \mathrm{~Hz}$. The results are compared with analytical results obtained with an equivalent periodically supported Timoshenko beams with supports spacing equal to the one of the left or right zones (formula from [5]). Analytical formulas and WFE results are in close agreement for points sufficiently far from the transition. Whereas the transition is steep, the rail needs several meters to pass from one mechanical behaviour to the other one. This transition does not show overcharged support.

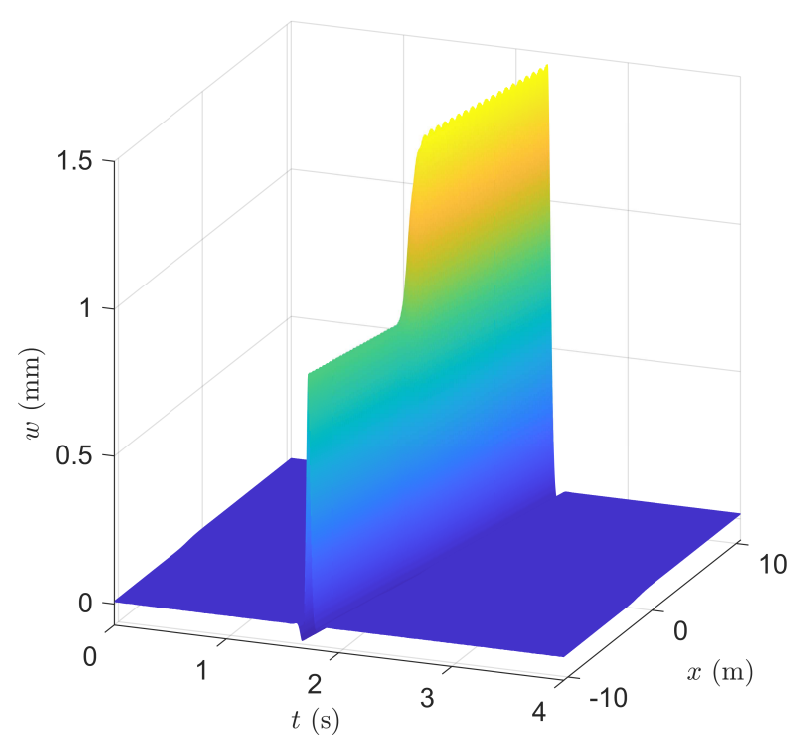

(a) Rail deflection against time and position.

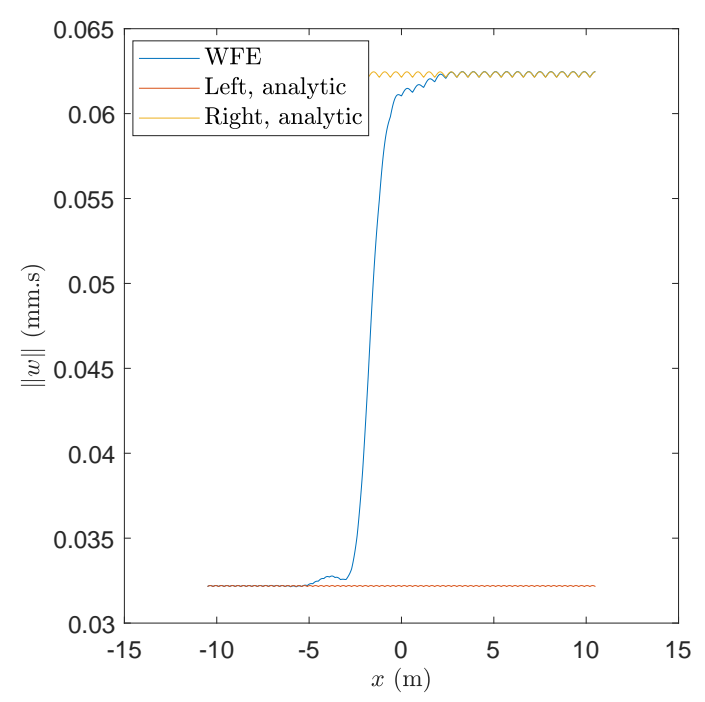

(b) Rail deflection in the frequency domain (at $5 \mathrm{~Hz}$ ) against position. WFE results compared with analytical results obtained for an infinite track.

Figure 3: Rail deflection for a variation in the support spacing.

\section{Conclusion}

The Wave Finite Element method is very efficient to compute infinite periodic structures mechanical behaviour under charges compared to classical finite element methods (see [18]). This article presented new developments to apply this method to transition zones between two semi-infinite periodic structures. Railway examples of application were presented and show a strong agreement with other semi-analytical methods or analytical laws. This method can also provide information on the damping of waves which can propagate over a given structure. 


\section{REFERENCES}

[1] P.M. Belotserkovskiy. On The Oscillation of infinite periodic beams subjected to a moving concentrated force. Journal of Sound and Vibration, 193(3):705-712, jun 1996.

[2] A. Nordborg. Vertical Rail Vibrations: Parametric Excitation. ACUSTICA - acta acustica, 84:289-300, 1998.

[3] G. Bonnet and R. Lassoued. Response of a Periodically Supported Beam To a Moving Load From the Superposition of Bloch Waves. In 7th European Conference on Structural Dynamics Eurodyn, Southampton, 2008.

[4] T. Hoang, D. Duhamel, G. Foret, H.P. P. Yin, P. Joyez, and R. Caby. Calculation of force distribution for a periodically supported beam subjected to moving loads. Journal of Sound and Vibration, 388:327-338, feb 2017.

[5] T. Hoang, D. Duhamel, and G. Foret. Dynamical response of a Timoshenko beams on periodical nonlinear supports subjected to moving forces. Engineering Structures, 176:673$680, \operatorname{dec} 2018$.

[6] A.V. Metrikine and K. Popp. Vibration of a periodically supported beam on an elastic half-space. European Journal of Mechanics - A/Solids, 18(4):679-701, jul 1999.

[7] E. Arlaud, S. Costa D'aguiar, and E. Balmes. Receptance of railway tracks at low frequency: Numerical and experimental approaches. Transportation Geotechnics, 9:1-16, 2016.

[8] S. Costa D’Aguiar, E. Arlaud, R. Potvin, E. Laurans, and C. Funfschilling. Railway Transitional Zones: A Case History from Ballasted to Ballastless Track. International Journal of Railway Technology, 3:37-61, 2014.

[9] E. Fortunato, A. Paixão, and R. Calçada. Railway Track Transition Zones: Design, Construction, Monitoring and Numerical Modelling. International Journal of Railway Technology, 2(4):33-58, 2013.

[10] H. Wang and V. L. Markine. Methodology for the comprehensive analysis of railway transition zones. Computers and Geotechnics, 99:64-79, jul 2018.

[11] T. Hoang, D. Duhamel, G. Foret, H.P. Yin, G. Cumunel, P. Joyez, and R. Caby. Response of a Periodically Supported Beam on a Non-Uniform Viscoelastic Foundation subject to Moving Loads. In J. Pombo, editor, Proceedings of the Third International Conference on Railway Technology: Research, Development and Maintenance, number April, Stirlingshire, Scotland, 2016. Civil-Comp Press.

[12] G. Kouroussis, D. P. Connolly, G. Alexandrou, and K. Vogiatzis. Railway ground vibrations induced by wheel and rail singular defects. Vehicle System Dynamics, 53(10):1500-1519, oct 2015.

[13] M. Germonpré, G. Degrande, and G. Lombaert. A track model for the prediction of groundborne vibrations due to parametric excitation. Procedia Engineering, 199:2663-2668, jan 2017. 
[14] M. Germonpré, G. Degrande, and G. Lombaert. Periodic track model for the prediction of railway induced vibration due to parametric excitation. Transportation Geotechnics, 17:98-108, dec 2018.

[15] E. Arlaud. Modèles dynamiques réduits de milieux périodiques par morceaux : application aux voies ferroviaires. PhD thesis, École Nationale Supérieure d'Arts et Métiers, 2016.

[16] J. M. Mencik and D. Duhamel. A wave-based model reduction technique for the description of the dynamic behavior of periodic structures involving arbitrary-shaped substructures and large-sized finite element models. Finite Elements in Analysis and Design, 101:1-14, 2015.

[17] T. Hoang, D. Duhamel, G. Foret, and Computing Methods. Wave finite element method for vibration of periodic structures subjected to external loads. In 6th European Conference on Computational Mechanics (ECCM 6), number June, pages 11-15, 2018.

[18] T. Hoang, D. Duhamel, G. Foret, J.L. Pochet, and F. Sabatier. Wave finite element method for the dynamic analysis of railway tracks. In 13th World Congress on Computational Mechanics (WCCM XIII) 2nd Pan American Congress on Computational Mechanics (PANACM II), New York City, NY, USA, 2018. 